[4] Stephen B, Rhian MD, Adam SB, Simon GT, and the EPIC-InterAct. Network Mendelian randomization: using genetic variants as instrumental variables to investigate mediation in causal pathways. Int J Epidemiol. 2015 Apr; 44(2): 484-495

Disclosure of Interests: None declared

DOI: 10.1136/annrheumdis-2021-eular.2061

\section{Immune events with checkpoint inhibitors}

\section{OP0194 GENERALIZED IMMUNE ACTIVATION IN STRUCTURES RELATED TO PMR OR GCA ON PET/CT ASSESSMENT DOES NOT OCCUR IN IMMUNE CHECKPOINT INHIBITOR-TREATED PATIENTS WHO DO NOT GO ON TO DEVELOP RHEUMATIC IMMUNE-RELATED ADVERSE EVENTS}

D. Liew ${ }^{1,2,3}$, A. Poon ${ }^{4}$, C. Mcmaster ${ }^{1,2,3}$, C. Owen ${ }^{1,3}$, J. Leung ${ }^{1,3}$, A. Frauman ${ }^{2,3}$, J. Cebon ${ }^{5,6,7}$, A. Scott ${ }^{3,4,5}$, R. Buchanan ${ }^{1,3}$. 'Austin Hospital, Rheumatology, Heidelberg, Australia; ${ }^{2}$ Austin Hospital, Clinical Pharmacology and Therapeutics, Heidelberg, Australia; ${ }^{3}$ University of Melbourne, Medicine, Parkville, Australia; ${ }^{4}$ Austin Hospital, Molecular Imaging and Therapy, Heidelberg, Australia; ${ }^{5}$ Olivia Newton-John Cancer Wellness \& Research Centre, Medical Oncology, Heidelberg, Australia; ${ }^{6}$ La Trobe University, Melbourne Campus, Tumour Immunology Laboratory, Bundoora, Australia; ${ }^{7}$ La Trobe University, Melbourne Campus, School of Cancer Medicine, Bundoora, Australia

Background: The pathogenesis of rheumatic immune-related adverse events (irAEs) from checkpoint inhibitor cancer immunotherapy directed against programmed cell death protein 1 (PD-1) and programmed death ligand 1 (PD-L1) remains unknown, even though they are a consequence of pharmacologic inhibition of a specific immune mechanism. Given that some irAEs resemble polymyalgia rheumatica (PMR) or giant cell arteritis (GCA), a disease whose pathogenesis is poorly understood, observations regarding the pathogenesis of PMR-like or GCA-like irAEs are of significant interest. One proposed pathogenic mechanism involves generalized immune activation leading to a spectrum of subclinical disease. Interrogation of this hypothesis may be aided by PET/CT, which is frequently utilized for oncological staging purposes but is also useful in classical PMR or GCA diagnosis. If PMR or GCA irAEs merely represent a spectrum of generalized immune activation, low-grade subclinical PMR or GCA-related changes on PET/ CT might be expected to be seen in patients who receive immunotherapy, irrespective of whether they develop clinically evident rheumatic irAEs.

Objectives: This study investigated whether such changes occurred in patients receiving immunotherapy who did not develop clinically evident rheumatic irAEs. Methods: Consecutive patients exposed to PD-1 or PD-L1 inhibitor immunotherapy at a single center had scintigraphic uptake calculated by a nuclear medicine physician experienced in assessment of vasculitis. Patients were included if they had had 18F-fluorodeoxyglucose (18F-FDG) PET/CT imaging both within the two weeks prior to immunotherapy initiation and after at least eleven weeks of immunotherapy. Patients who went on to develop a rheumatic irAE were excluded, as were patients with scintigraphic evidence of liver metastases owing to their potential influence on scoring of uptake. Quantification of 18F-FDG uptake by maximum standardized uptake values (maximum standard unit value, SUVmax) was performed at sites relevant to PMR or GCA (17 sites relevant to PMR, 17 sites relevant to GCA) in paired scans, and the difference calculated.

Results: Twenty-four patients receiving nivolumab, pembrolizumab or avelumab met the inclusion criteria, primarily for melanoma, non-small cell lung cancer, or lymphoma. The mean age was 67 at the time of the first scan, $71 \%$ were male, and $66 \%$ had a complete or partial oncological response at best response. No statistically or clinically significant difference in SUVmax was noted at any PMR or GCA-relevant anatomical site interrogated. Latent class analysis did not reveal clusters identifiable by cancer type, best response, or presence of combination therapy.

Conclusion: Patients treated with PD-1/PD-L1 inhibitors without clinically evident rheumatic irAEs do not develop subclinical PMR or GCA-like changes on $\mathrm{PET} / \mathrm{CT}$. This supports the proposition that PMR-like and GCA-like irAEs are a distinct entity with stochastic onset, and do not simply represent generalized immune activation induced by immunotherapy.

Acknowledgements: David Liew is the recipient of the Ronald John Gleghorn Bursary from the University of Melbourne.

Disclosure of Interests: David Liew: None declared, Aurora Poon: None declared, Christopher McMaster: None declared, Claire Owen Speakers bureau: Roche, Jessica Leung Speakers bureau: Gllead, Novartis, Albert Frauman: None declared, Jonathan Cebon: None declared, Andrew Scott: None declared, Russell Buchanan: None declared

DOI: 10.1136/annrheumdis-2021-eular.2788

\section{OP0195 \\ PLASMA CHECKPOINT PROTEIN LEVELS AND GALECTIN-9 IN JUVENILE SYSTEMIC LUPUS ERYTHEMATOSUS}

E. Sag ${ }^{1,2}$, K. Yüksel ${ }^{1}$, S. Demir ${ }^{1}$, S. Ozdel ${ }^{3}$, U. Kaya Akca ${ }^{1}$, E. Atalay ${ }^{1}$, M. Kasap-Cuceoglu ${ }^{1}$, R. Topaloglu ${ }^{4}$, Y. Bilginer ${ }^{1,2}$, S. Özen ${ }^{1,2} .{ }^{1}$ Hacettepe $^{2}$ University Faculty of Medicine, Department of Pediatric Rheumatology, Ankara, Turkey; ${ }^{2}$ Hacettepe University Faculty of Medicine, Translational Medicine Laboratories, Pediatric Rheumatology Unit, Ankara, Turkey; ${ }^{3} \mathrm{Dr}$ Sami Ulus Maternity and Child Health and Diseases Training and Research Hospital, Department of Pediatric Rheumatology, Ankara, Turkey; ${ }^{4}$ Hacettepe University Faculty of Medicine, Department of Pediatric Nephrology, Ankara, Turkey

Background: Systemic Lupus Erythematosus (SLE) is the prototype for a systemic autoimmune disease. SLE is a disease of the adaptive immune system where $T$ lymphocyte dysfunction has an important role as well. Checkpoint proteins have become an important topic in the study of Tcell. The balance of $\mathrm{T}$ cell co-stimulatory and co-inhibitory receptors is important for the regulation of the adaptive immune system response.

Objectives: In this study we aimed to assess the checkpoint proteins in childhood SLE patients

Methods: Fourty-nine jSLE patients together with 15 age- and gendermatched controls were included. Clinical features, disease activity scores and laboratory parameters were recorded both retrospectively and at the time of samplimg. Patient samples were collected at their last visit, Plasma CD25 (IL-2R $\alpha$ ), 4-1BB, B7.2 (CD86), TGF- $\beta 1$, CTLA-4, PD-L1, PD-1, Tim-3, LAG- 3, Galectin-9 levels were studied by the cytometric bead-based multiplex assay panel according to manufacturer's instruction (LEGENDplex HU Immune Checkpoint Panel 1 (10-plex); catalogue number 740962, Biolegend) and analysed by Novocyte 3005 flow cytometer. Two-step cluster analysis procedure was conducted over the chosen 7 symptom status and 3 clusters were chosen for the final analysis

Results: A total of 49 patients ( $71.4 \%$ female) diagnosed with SLE according to the SLICC criteria and 15 healthy controls $(73.3 \%$ female) were included in the study. The mean age of the patients was $17.7 \pm 2.6$ years and the controls was $13.3 \pm 1.4$ years. The median disease duration was 5.7 years. At the time of sampling fifteen of the patients had a SLEDAl score of zero (no activity), 21 of them had between 1-5 (mild activity), 7 of them had between 6-10 (moderate activity), 5 of them had between 11-19 (high activity), 1 of them had >20 (very high activity)

Galectin-9 and PD-L1 were significantly higher in SLE patients. Other checkpoint proteins and IL-2R $\alpha$ were also higher but did not reach statistical significance. There were significant correlations between SLEDAI and IL-2R $\alpha$, Galectin-9 and PDL1. (Figure 1) There were three clinical clusters: Cluster 1 included patients with no major organ involvement, cluster 2 had predominantly haematological involvement $(n=16)$ and cluster $3(n=11)$ had predominantly renal involvement. Checkpoint proteins were not different among these three clusters.
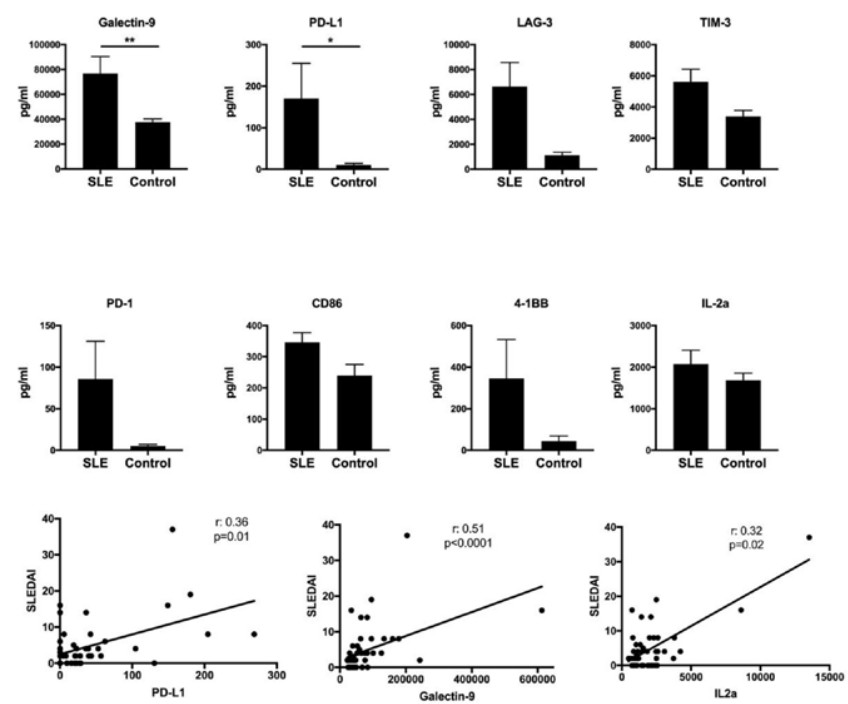

Figure 1. 